\title{
Natural ultrasonic echoes from wing beating insects are encoded by collicular neurons in the CF-FM bat, Rhinolophus ferrumequinum
}

\author{
Gerd Schuller \\ Zoologisches Institut der Ludwig-Maximilians-Universität München, Luisenstrasse 14, D-8000 München 2, \\ Federal Republic of Germany \\ Accepted March 23, 1984
}

\begin{abstract}
Summary. 1. Acoustic reflections from a wing beating moth to an $80 \mathrm{kHz}$ ultrasonic signal were recorded from six different incident angles and analyzed in spectral and time domains. The recorded echoes as well as independent components of amplitude and frequency modulations of the echoes were employed as acoustic stimuli during single unit studies.

2. The responses of single inferior colliculus neurons to these stimuli were recorded from four horseshoe bats, Rhinolophus ferrumequinum, a species which uses a long constant frequency (CF) sound with a final frequency modulated (FM) sweep during echolocation. All neurons responding to wing beat echoes reliably encoded the fundamental wing beat frequency as well as the more refined frequency and amplitude modulations.

3. These neurons may provide the bat a neural mechanism to detect periodically moving targets against a cluttered background and also to discriminate various insect species on the basis of their wing beat patterns.
\end{abstract}

\section{Introduction}

The greater horseshoe bat belongs to the group of so-called CF-FM-bats which employ an echolocation pulse consisting of a long constant frequency component (CF) terminated by a short downward frequency sweep (FM).

The duration of the CF component is typically between 10 and $60 \mathrm{~ms}$ which can be lengthened up to $100 \mathrm{~ms}$ when the animal is confronted with

Abbreviations: $C F$ constant frequency; $A M$ amplitude modulation; $F M$ frequency modulation; $S A M$ sinusoidal amplitude modulation; $S F M$ sinusoidal frequency modulation; $B F$ best frequency; FFT Fast Fourier Transform difficult detection tasks (Schnitzler and Flieger 1983). The frequency of the CF component is remarkably constant with a variance of less than $100 \mathrm{~Hz}$. The frequency of consecutive sounds is kept with similar precision at the resting frequency when the bat is stationary or stabilized accurately at a lowered frequency when the bat compensates for Doppler shifts (Schuller et al. 1974).

The constant frequency component of the echolocation sound is highly sensitive to movements of the targets which introduce small frequency modulations due to the Doppler effect. For example, wing beats of insects (moths of small size) modulate the $61 \mathrm{kHz}$ CF component of the echolocation sound with a modulation depth of $800 \mathrm{~Hz}$ in Pteronotus p. parnellii (Goldman and Henson 1977 ) or up to $3 \mathrm{kHz}$ on the $80 \mathrm{kHz}$ CF component of rhinolophids (Schnitzler et al. 1983).

It has been postulated that long CF-FM bats, including the greater horseshoe bat, can detect such periodical modulations of the CF component produced by wing beats to discriminate various insect species (Pye 1967; Schnitzler 1970; Neuweiler et al. 1980).

Evoked potential recordings from the inferior colliculus of horseshoe bats showed that frequency modulation depth from $10 \mathrm{~Hz}$ to a few $\mathrm{kHz}$ or a rate of frequency change larger than $6 \mathrm{~Hz}$ per ms elicited pronounced neural responses (Schuller 1972). Behaviorally, Goldman and Henson (1977) demonstrated in Pteronotus $p$. parnellii, and Trappe (1982) in the greater horseshoe bat that these CF-FM bats would capture wing beating insects or attack a 'wing beating' model but would remain uninterested in the same targets when their wings were not beating. Single unit studies in the inferior colliculus and cochlear nucleus of the horseshoe bat (Schuller 1979; Pollak and Schuller 1981; Vater 1982), and the auditory cortex (Ost- 
wald 1980) further showed that the auditory system of the horseshoe bat is highly sensitive to small sinusoidal modulations of pure tones in a wide range of modulation frequencies, suggesting that the system can encode the fine structure of wing beat echoes. This postulation, however, must be considered tenuous since the actual wing beat reflections are considerably more complicated than the sinusoidal modulations (Schnitzler et al. 1983). In this study natural wing beat reflections from a moth were employed as stimuli while recording from single collicular neurons in order to obtain a fuller understanding of how wing beats might be analyzed and encoded within the bat's brain.

\section{Methods and materials}

Four greater horseshoe bats, Rhinolophus ferrumequinum, were employed in this study. The anaesthetized animals were surgically prepared for recordings as described by Schuller (1979). The activity of single neurons was recorded in awake or slightly sedated (less than half of the normal pentobarbital dose) animals with conventional methods using $3 \mathrm{~mol} / \mathrm{l} \mathrm{KCl}$ filled glass pipettes. Alcian blue dye was added to the electrolyte (Harnischfeger 1979) for later histological verification of the recording sites.

Echoes from the beating wings were obtained from a moth, Noctua pronuba, a very common noctuid with a body length of $26-29 \mathrm{~mm}$ and a maximum wing span of $40-50 \mathrm{~mm}$. Such moths are preyed upon by the Indian rhinolophid, Rhinolophus rouxi (Eckrich, personal communication) and presumably also by European rhinolophids. The thorax of a moth was tethered to a small clamp and in such a position that the moth would continuously beat its wings. An $80 \mathrm{kHz}$ ultrasonic tone was broadcasted from a loudspeaker towards the moth and the reflections were picked up by a Brüel \& Kjaer 1/4 inch microphone placed beneath the loudspeaker and they were stored on magnetic tape (Philipps, Analog 7). Reflections from six different relative orientation angles were recorded.

From these recorded sounds three different stimulus versions were derived: 1) the original echoes, 2) pure frequency modulated (FM) versions of the original echoes, i.c., reflections without amplitude modulations and 3) pure amplitude modulated (AM) versions of the original echoes, i.e., reflections without frequency modulations. To obtain the FM version of an echo, the recorded echo was passed through a zero crossing comparator, which controlled a phase locked generator (Wavetek 186) to produce the constant amplitude replica of the wing beat reflection. The AM version was derived from feeding the original waveform through a double phase rectifier circuit controlling the VCA input of a Wavetek 136 generator at the carrier frequency. These three continuous waveform signals were gated to produce stimuli with a $100 \mathrm{~ms}$ duration and a $2 \mathrm{~ms}$ rise and fall time approximating the temporal parameters of natural wing beat reflections. Individual stimuli were presented at a rate of $3.4 \mathrm{~Hz}$, corresponding to the mean rate used by the bat during the search phase. The spectro-temporal composition of the stimuli was determined with Fast Fourier Transform (FFT) procedures on a computer (Digital Equipment Corp. PDP 11/23).

The analysis of the wing beat stimulus responses was restricted mainly to neurons with best frequencies around the $\mathrm{CF}$ frequency of each individual bat. Since the best frequencies of these neurons varied slightly, the carrier frequencies of the stimuli were adjusted to the neuron's best frequency (BF) or another desired carrier frequency by the double heterodyne mixing method described in detail in Schuller et al. (1974). The peak intensities of the wing beat stimuli were monitored and they ranged from 40 to $70 \mathrm{~dB}$ SPL and were typically 30 to $50 \mathrm{~dB}$ above the response thresholds at unit's respective BFs. General characteristics of each neuron were measured with pure tones, sinusoidally frequency modulated (SFM) stimuli (modulation rate: $30 \mathrm{~Hz}$, modulation depth: $50 \mathrm{~Hz}$ to $2 \mathrm{kHz}$ ) and with sinusoidally amplitude modulated (SAM) stimuli (modulation rate: $30 \mathrm{~Hz}$ and modulation height: $100 \%$ or less). The unit's responses were processed by the PDP-11 computer and presented in the form of post-stimulus-time histograms deriving from 30 presentations of stimuli.

\section{Results}

\section{Spectral composition of wing beat reflections}

All recorded echoes showed distinct amplitude and frequency modulations at about $30 \mathrm{~Hz}$, the fundamental beat frequency of the moth wings (Fig. 1). Additionally, a complicated pattern of frequency and amplitude modulation was superimposed on each beat cycle. This complex acoustical pattern varied with the direction from which the reflections were recorded (see also Schnitzler et al. 1983).

These results are expected since a moving wing, even simplified to a linear rigid plane moving periodically back and forth around a fixed axis, does not yield simple modulations of amplitude or frequency. For such a simple case, the effective reflection surface changes, to a first approximation, as a function of the angle between the impinging sound and the axis normal to the wing surface. The echo amplitude is proportional to the cosine function of time and angular velocity of the wing. Since the angular velocity is not constant the amplitude modulation is therefore a complicated function of time. The echo frequency is modulated in an even more complicated manner because frequency modulation is velocity dependent and therefore is a function of the distance of the reflecting surface from the rotating axis.

The temporal distribution of spectral energy of such echoes is shown in Fig. 1. In each graph the spectral energy of the echo (upper) and its amplitude envelope (lower) is depicted as a function of time for each of six incident angles. The arrows in the pictograms to the left indicate the directions from which the insect was acoustically probed. The bars represent the band of frequencies at a given time ( 1 ms slices) which contains energy above an arbitrary fixed level (well above the noise level and $6 \mathrm{~dB}$ below the peak energy). This representation was chosen to avoid visual confusion resulting from the representation of the entire spectrum for each ms of the stimulus.

The prominence of amplitude or frequency modu- 

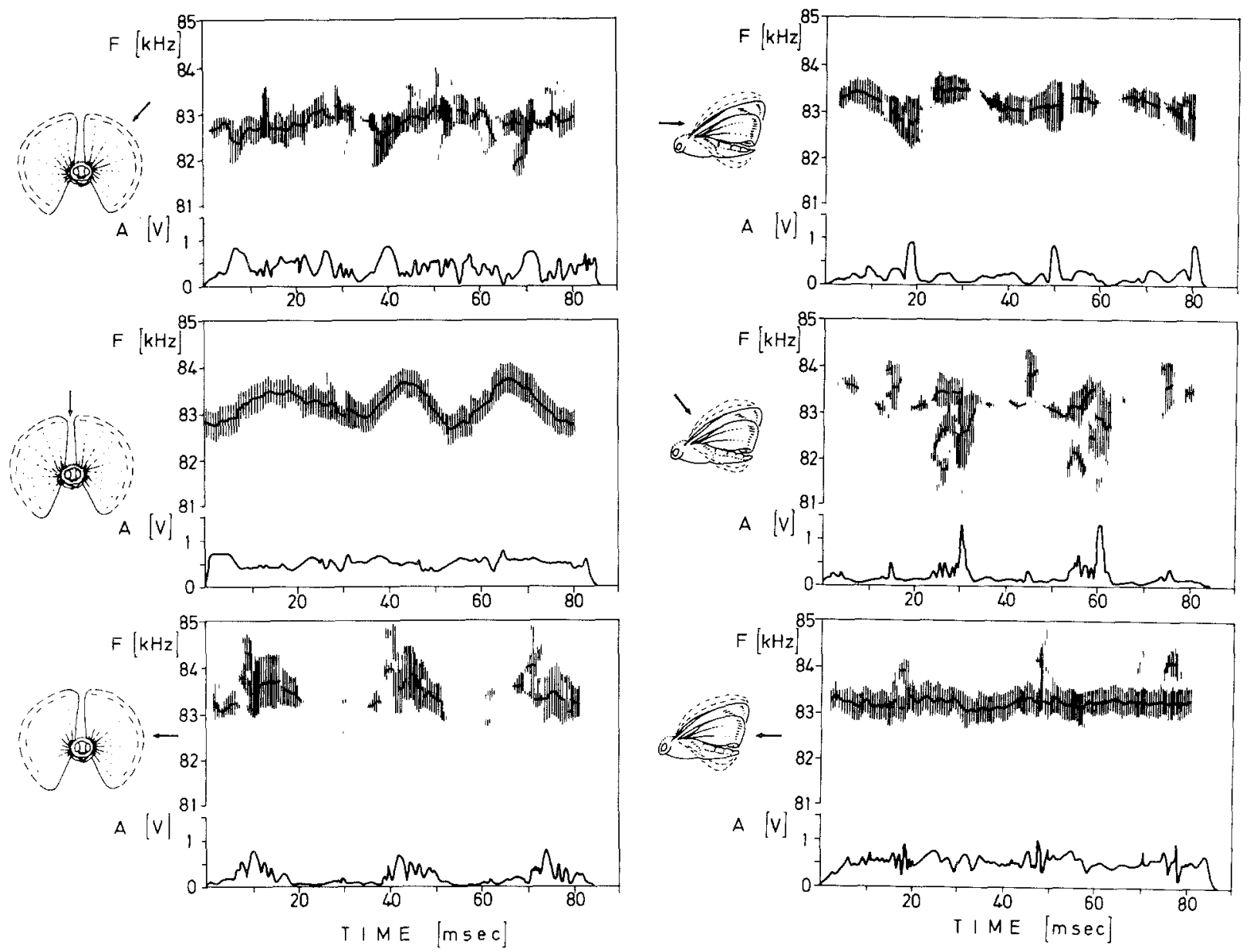

Fig. 1. Spectral composition of wing beat reflections. Left pictogram indicates from which direction the insect was acoustically irradiated. Wing beat reflections were recorded from the same direction (arrow). The upper part of each graph shows the spectral energy of the reflection as a function of time (abscissa). Dark line marks the peaks of spectral energy, whereas vertical bars indicate the frequency range in which the signal energy was above an arbitrarily fixed level (about $6 \mathrm{~dB}$ below the peak energy). The curve below gives the amplitude function (envelope) of wing beat reflections. Note that the amplitude function is highly structured in the lower left and the 2 upper right graphs, whereas the strongest frequency deviations occur in the middle left reflection

lations varied according to sound incidence. The reflections from above (1st column, middle) and from behind the moth ( $2 \mathrm{~d}$ column, bottom) showed small amplitude modulations, whereas, frontal reflections pronounced amplitude peaks ( $2 \mathrm{~d}$ column, middle and top). Frequency modulation was prominent in almost all echoes except those reflected from behind the insect ( $2 \mathrm{~d}$ column, bottom) where the frequency excursions were very small and short.

\section{Basic properties of the recorded neurons}

Thirty nine of a total of 157 neurons recorded from the inferior colliculus (central nucleus) were tested with wing beat modulated stimuli. Complete tests of all available wing beat stimuli and at different carrier frequencies and intensities were obtained in 27 neurons. The best frequencies of all but one cell ranged from $75.4 \mathrm{kHz}$ to $85.0 \mathrm{kHz}$ and most $(72 \%)$ were be- tween 82 and $84 \mathrm{kHz}$ around the frequencies of the CF components of echolocation sounds of these bats. The Q-10dB values of these neurons were high and ranged from 54 to 276 . One low frequency neuron with a best frequency of $57 \mathrm{kHz}$ was included in the study as it also responded to wing beat stimuli at a carrier frequency of $83 \mathrm{kHz}$. This neuron was very broadly tuned with a Q-10dB value of 2.2 . Thirteen of the 39 neurons displayed a phasic discharge pattern to pure tones whereas the rest showed a tonic or sustained response pattern. The general response properties of the neurons to SFM and SAM stimuli were similar to those reported in detail by Pollak and Schuller (1981).

\section{Responses of neurons to wing beat reflections}

The majority of neurons showed synchronized responses to either all echo stimuli deriving from differ- 


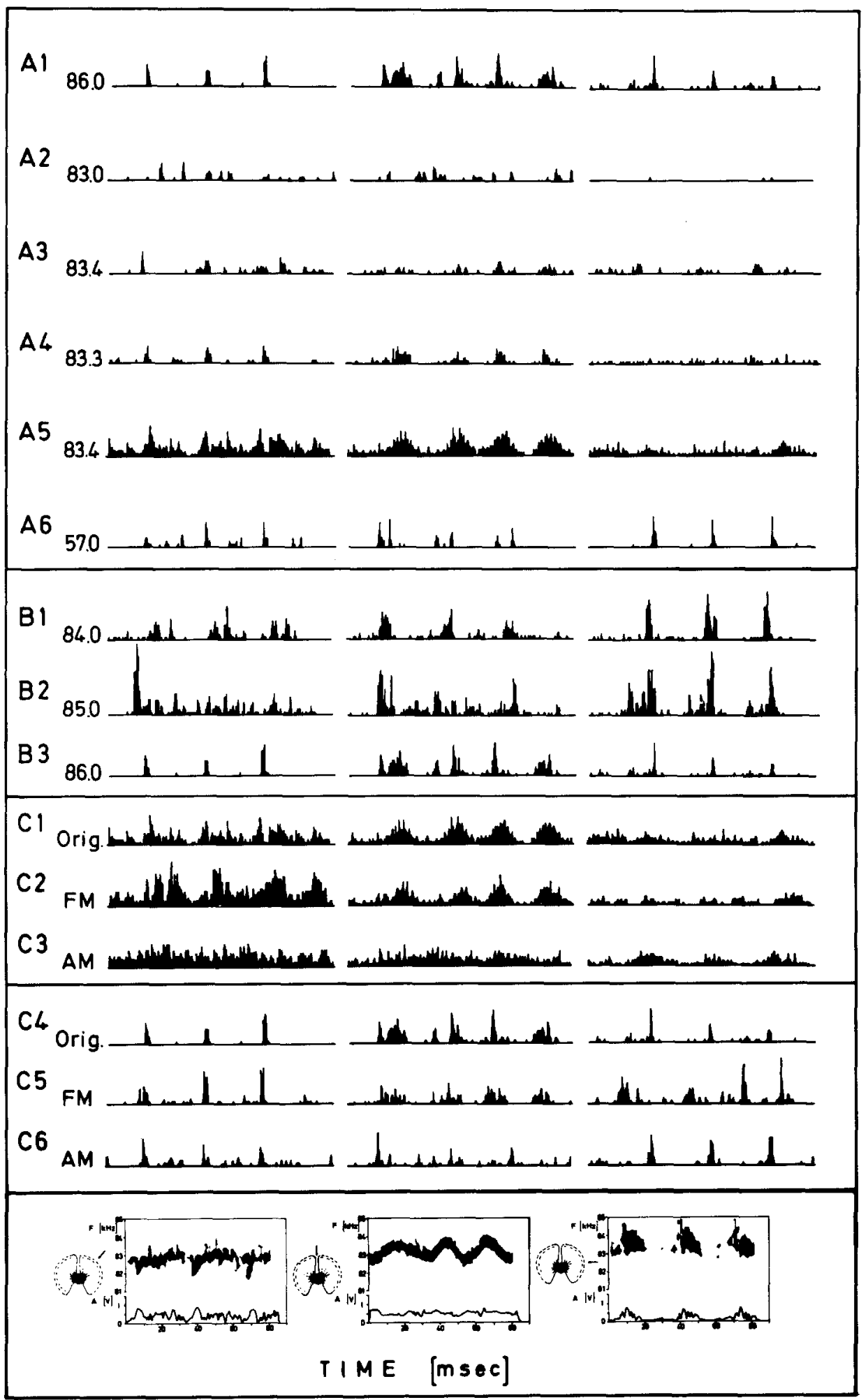

Fig. 2. a Neuronal responses to wing beat reflections. A1 through A6: responses of 6 different neurons. B1 through B3: responses of a single neuron to reflections at different carrier frequencies. $\mathrm{C} 1$ to $\mathrm{C} 3$ and $\mathrm{C} 4$ to $\mathrm{C} 6$ : response patterns of two neurons to the original reflections as compared to the isolated FM and AM components. The lower part depicts the stimuli used for eliciting neuronal responses shown in the corresponding column 


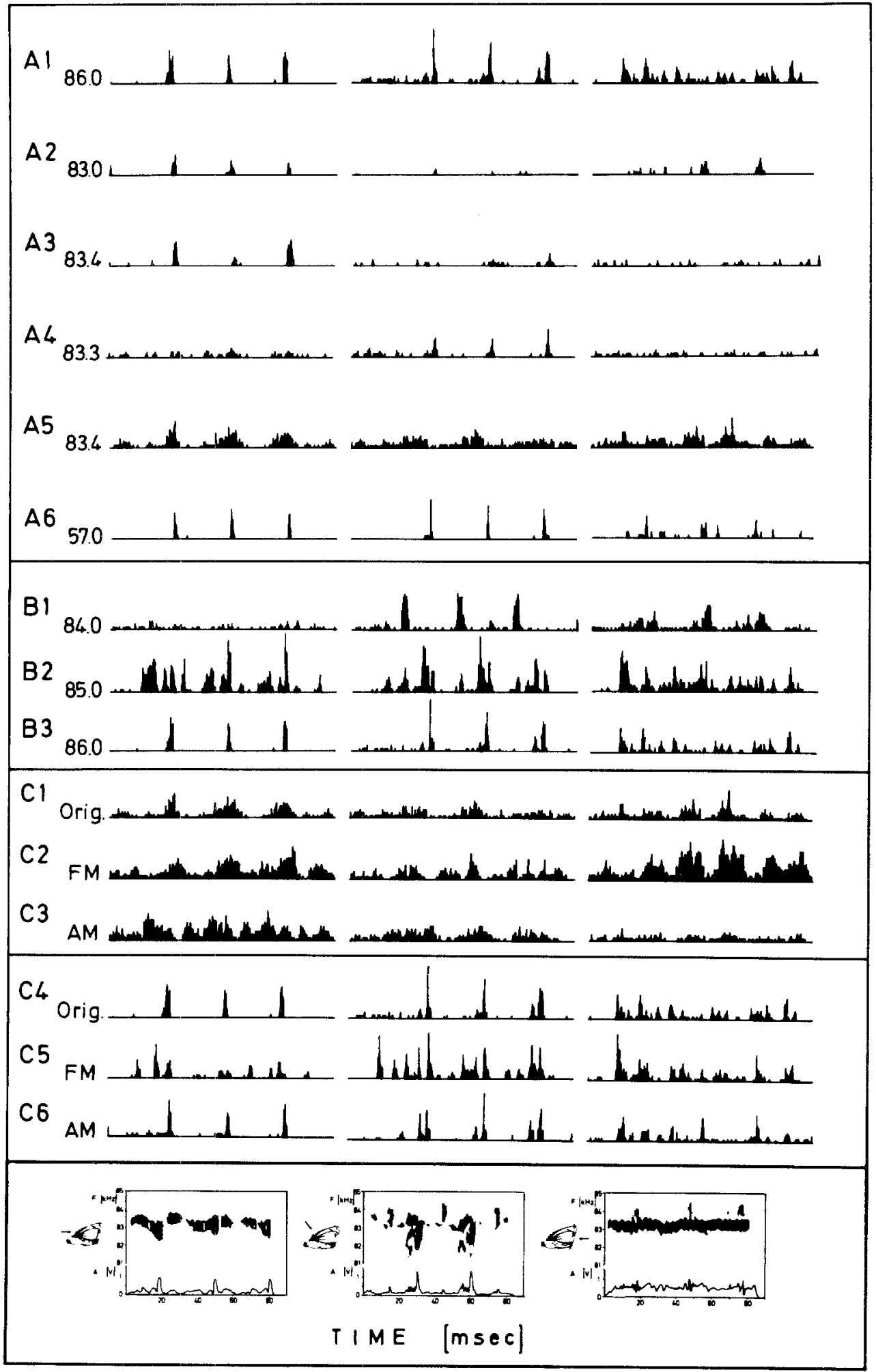

Fig. 2. b Continuation of Fig. 2 a; see legend of Fig. 2 a 
Table 1. Characteristics of neurons represented in Fig. $2 \mathrm{a}$ and $\mathrm{b}$

\begin{tabular}{|c|c|c|c|c|c|c|c|}
\hline \multirow{3}{*}{$\begin{array}{l}\text { Label } \\
\text { Fig. } 2\end{array}$} & \multirow{3}{*}{$\begin{array}{l}\text { Unit } \\
\text { No. }\end{array}$} & \multicolumn{3}{|c|}{ Stimulus } & \multirow{3}{*}{$\begin{array}{l}\mathrm{BF} \\
(\mathrm{kHz})\end{array}$} & \multicolumn{2}{|c|}{$\begin{array}{l}\text { Synchroni- } \\
\text { zation }\end{array}$} \\
\hline & & \multirow{2}{*}{$\begin{array}{l}f_{\text {carr }} \\
(\mathrm{kHz})\end{array}$} & \multirow{2}{*}{$\begin{array}{l}\text { Int } \\
\text { (SPL) } \\
\text { (dB) }\end{array}$} & \multirow{2}{*}{ Type } & & & \\
\hline & & & & & & SAM & SFM \\
\hline A1 & 16 & 86.0 & $40 / 30$ & $\mathrm{O}$ & 85.0 & ++ & $+t$ \\
\hline $\mathrm{A} 2$ & 14 & 83.0 & $70 / 20$ & $\mathrm{O}$ & 57.5 & $+t$ & - \\
\hline $\mathrm{A} 3$ & 6 & 83.4 & $60 / 32$ & $\mathrm{O}$ & 83.2 & + & + \\
\hline A4 & 7 & 83.3 & $60 / 30$ & $\mathrm{O}$ & 83.3 & + & ++ \\
\hline A5 & 5 & 83.4 & $60 / 40$ & $\mathrm{O}$ & 82.7 & + & ++ \\
\hline A6 & 14 & 57.0 & $60 / 30$ & $\mathrm{O}$ & 57.5 & ++ & - \\
\hline B1 & 16 & 84.0 & $40 / 30$ & $\mathrm{O}$ & 85.0 & ++ & ++ \\
\hline B2 & & 85.0 & & $\mathrm{O}$ & & & \\
\hline B3 & & 86.0 & & $\mathrm{O}$ & & & \\
\hline $\mathrm{C} 1$ & 5 & 83.4 & $60 / 40$ & $\mathrm{O}$ & 82.7 & + & $+t$ \\
\hline $\mathrm{C} 2$ & & & & FM & & & \\
\hline $\mathrm{C} 3$ & & & & $\mathrm{AM}$ & & & \\
\hline $\mathrm{C} 4$ & 16 & 86.0 & $40 / 30$ & $\mathrm{O}$ & 85.0 & ++ & ++ \\
\hline $\mathrm{C} 5$ & & & & $\mathrm{FM}$ & & & \\
\hline C6 & & & & AM & & & \\
\hline
\end{tabular}

+ synchronization to modulation; ++ strong synchronization to modulation; - no synchronization; the intensity indication after the slash $(/)$ indicates the superthreshold intensity. $O$ original wing beat reflection; $F M$ frequency modulated component of the wing beat stimulus; $A M$ amplitude modulated component of the wing beat stimulus

ent incident angles or to a few of these stimuli (Figs. $2 \mathrm{a}$ and $2 \mathrm{~b}$ ). The discharge patterns were in general complex as were the stimuli. Figures $2 \mathrm{a}$ and $2 \mathrm{~b}$ show in section A (A1 to A6) responses of individual neurons to the 6 different wing beat reflections. A1 represents a neuron that responded synchronously to all types of wing beat reflections at a carrier frequency of $86 \mathrm{kHz}(1 \mathrm{kHz}$ above BF). Other neurons (A2-A5) showed synchronized discharges to a smaller subset of signals. In neuron A3, for example, the synchronization to one type of reflection (echo from ahead of the insect, Fig. 2b) was prominent but not to others. Neuron A6 which had a low BF $(57 \mathrm{kHz})$, responded synchronously to wing beat modulations superimposed on its BF. These data suggest that synchronized responses to wing beat stimuli are probably a general property of neurons in the inferior colliculus and not unique for neurons with BFs in the $80 \mathrm{kHz}$ range. Eight $(20 \%)$ neurons showed no or only weak synchronization to wing beat modulations. Interestingly, these cells also did not produce good synchronized responses to SFM or SAM stimuli. The basic properties of the presented neurons and additional information on stimulus parameters are summarized in Table 1.

The synchronous activities of the IC neurons could be attributed to either the FM and AM components of the complex stimuli, or both. In order to examine these possibilities, the FM and AM versions of the stimuli were presented to the animal following the stimulation with natural echoes. Two classes of response types could be distinguished.

In one class the neuron's responses to the natural wing beat reflections were congruent with the responses to one component, i.e. the FM or AM component, for all six wing beat stimuli. Section $C$ of Figs. $2 a$ and $2 b$ illustrates the responses of two neurons which belonged to this first class. Responses shown in the upper lines ( $\mathrm{C} 1$ and $\mathrm{C} 4)$ are those to the original wing beat echoes. The responses of the first neuron to the FM component alone (C2) matched closely the responses to the natural echoes, whereas the AM stimuli elicited poor synchronized responses (C3). Such neurons have been classified as FM-governed.

Responses of a different neuron ( $\mathrm{C} 4$ to $\mathrm{C} 6)$ were synchronized to all three stimulus forms (natural, FM, AM). However, the synchrony to the wing beat echoes was clearly more similar to the responses to the AM components than to the FM components. Such neurons have been called AM-governed. The response patterns of such neurons to the FM components were more intricate in comparison with the discharges to the other two stimuli.

Neurons within this first class, AM or FM-governed, also showed predominant activity to either SAM or SFM stimulation. All units which showed well synchronized responses to SFM but not to SAM were generally FM-governed, whereas those which displayed tightly synchronized responses to SAM were generally AM-governed even though the latter units might respond synchronously to SFM as well.

The majority of IC neurons could not be classified simply as FM- or AM-governed, since responses of a neuron in this category (class II) to the six wing beat echoes were not always dominated by the frequency or amplitude modulation component. As the prominence of the two modulations (AM or FM) was different in individual wing beat echoes most neurons of this class also responded differentially by synchronizing to the stronger of the two components of modulation. Often the responses to the separate components were rather complicated, whereas the response to the combined natural signal exhibited sharper and more distinct peaks.

When tested with SFM and SAM stimuli these neurons exhibited in general synchronization to both modulation types, i.e., SFM and SAM were about equally effective in eliciting synchronized responses. The responses to natural wing beat echoes were consequently determined by the ratio of AM and FM modulation strength in the individual stimulus type. A change in the carrier frequency could either modify 
the degree of synchronization of the responses or alter the response patterns of a neuron drastically as shown in section B1 to B3 of Figs. $2 a$ and $2 b$. B1 to B3 show responses of a neuron at 3 different carrier frequencies, i.e., 84,85 and $86 \mathrm{kHz}$ respectively. A change of response synchronization is evident in Fig. 2 a, first column and Fig. 2b, first and third columns and a change in the latency of the response peaks is depicted in Fig. 2a, second column and Fig. 2b, second column. These changes are indifferent from those reported for IC neurons to a change in the carrier frequency of sinusoidally frequency modulated stimuli (Schuller 1979). The overall intensity of the stimuli was not systematically varied in this study. Changes of intensity within a range of $30 \mathrm{~dB}$ around the chosen level of $60 \mathrm{~dB}$ SPL did not reveal marked differences of response type but only influenced the overail activity of the neurons.

\section{Discussion}

The results of physical analysis clearly show that echoes from a natural moving target are complex and that certain features of the echoes are directionally dependent while others are directionally independent. Before discussing these data, some remarks about the natural behaviour of the greater horseshoe bats and their physical environment are in order. The horseshoe bat generally hunts for insects near or within trees or bushes. This dense reflecting background distorts and modulates the spectral composition of the CF-FM sounds of the bats. How can a bat, then, discriminate insects from vegetative background materials, or stationary from flying insects? In the following discussion only information deriving from the CF component will be considered.

The stationary targets will primarily attenuate the sound depending on the effective reflection surface of the target. When the bat approaches the target the frequency of the reflected echoes will be shifted upward due to the Doppler effect. The moving tree branches and leaves, due to the changes in the effective reflection surfaces of the targets (i.e. rotation by $90^{\circ}$ ), generate pronounced amplitude modulations of the echolocation sound. Additonally the movements of the objects produce further frequency modulations of the sounds, which tend to be of low magnitudes. The time course of such amplitude and frequency modulations is random and furthermore the modulation rate tends to be low.

The periodically moving wings of a flying insect produce cyclical modulations of frequency and amplitude in the echo, which are drastically different from those eminating from the cluttered background. Behavioural studies (Goldman and Henson 1977;
Vogler and Neuweiler 1983) have shown that CF-FM bats, Pteronotus p. parnellii or Rhinolophus ferrumequinum, only attacked and caught insects if the prey beat their wings but not when they sit still. These results suggest that periodic modulations are an important cue for target detection and discrimination. The data presented here and a recent study of Schnitzler et al. (1983) reveal that echoes from a wing beating insect show modulations of the carrier frequency up to several $\mathrm{kHz}$ and of amplitude up to almost $100 \%$. Furthermore, the magnitudes of amplitude and frequency modulations and their relative dominance are highly dependent on the direction of incidence of the sound (Fig. 1). This presents the bat with an additional problem for discrimination. During the pursuit of an insect the bat's relative position to the insect will continuously change so that the acoustical images of the insect heard by the bat will also change dynamically.

One feature of the echoes from wing beating insects, however, is independent of sound direction and is represented in virtually all modulation patterns, either as amplitude or frequency modulation: namely that of the fundamental wing beat frequency.

All neurons of the inferior colliculus sampled in this study reliably encoded the fundamental beat frequency of the wings regardless of the different echo types representing various sound directions. Moreover, this information was preserved in a neuron's responses even when the intensity or carrier frequency of the stimulus was changed within the unit's response area. Nevertheless, wing beat frequency is not a unique signature of a specific insect species. Consequently, discriminations of different insect species require processings of other more detailed information.

Indeed, the present recordings show that besides the periodicity of the movement the finer details of the spectral modulation pattern of the echoes are also encoded. The complex discharge patterns of many IC neurons closely corresponded to the fast frequency transitions. Despite such encoding capabilities, in no case did the recorded IC neurons discharge exclusively to only one specific modulation pattern. In fact, the responses to the complex stimuli could be explained on the basis of the neuron's responses to amplitude and/or frequency modulations or simulated sinusoidal AM or FM. Feature detection by single neurons seems therefore highly improbable at this level of signal analysis.

It is apparent that at the level of the inferior colliculus the highly structured acoustical patterns of echoes are preserved to a large extent in the neural response patterns. Little information is lost and specific modulation characteristics are even enhanced in some neural responses. Through these mechanisms 
the bat obtains a neural replica of the complex acoustic echoes associated with an individual insect. In a typical encounter, after the bat has detected the target, the pulse emission rate is considerably raised to a quasi continuous $\mathrm{CF}$ echolocation sound with duty cycles up to more than $90 \%$ (Vogler and Neuweiler 1983). In this way the bat increases the information flow considerably and thus can monitor the temporal development of the modulation pattern produced by the moving and turning insect. This information is analyzed in parallel by many IC neurons with adjacent BFs and these neurons have different sensitivities to the different constituents of the complex modulations. The information on the acoustical wing beat pattern of an insect is consequently present in the nervous system as a temporal sequency of complex response patterns.

It is possible that such temporal sequences of echoes and associated neural responses provide the identity of the insect species.

Acknowledgements: This work was supported by the Deutsche Forschungsgemeinschaft SFB 45 (TP B22) and SFB 204 (TP 10). The encouragement and suggestions for the manuscript of A. Feng and G. Neuweiler are gratefully acknowledged. I am indebted to H. Zöller for the PSTH-program and $\mathrm{K}$. Beuter for the FFT-routines.

\section{References}

Goldman LJ, Henson OW (1977) Prey recognition and selection by the constant frequency bat, Pteronotus $p$. parnellii. Behav Ecol Sociobiol 2:411-419

Harnischfeger G (1979) An improved method for extracellular marking of electrode tip positions in nervous tissues. J Neurosci Methods 1:195-200

Neuweiler G, Bruns V, Schuller G (1980) Ears adapted for the detection of motion, or how echolocating bats have exploited the capacities of the mammalian auditory system. J Acoust Soc Am 68:741-753

Ostwald J (1980) The functional organization of the auditory cortex in the CF-FM bat Rhinolophus ferrumequinum. In: Busnel RG, Fish JF (eds) Animal sonar systems. Plenum Press, New York London, pp 953-955
Pollak GD, Schuller G (1981) Tonotopic organization and encoding features of single units in inferior colliculus of horseshoe bats: functional implications for prey identification. J Neurophysiol $45: 208-226$

Pye JD (1967) Theories of sonar systems and their application to biological organisms (discussion) In: Busnel RG (ed) Animal sonar systems. Lab Physiol Acoust, CNRS Jouy-enJosas, France, pp 1121-1136

Schnitzler HU (1968) Die Ultraschall-Ortungslaute der Hufeisen-Fleđermäuse in verschiedenen Orientierungssituationen. Z Verg1 Physiol 57:376-408

Schnitzler HU (1970) Comparison of the echolocation behaviour in Rhinolophus ferrumequinum and Chilonycteris rubiginosa. Bijdr Dierk 40:77-80

Schnitzler HU (1978) Detection of movements by echolocating bats. Verh Dtsch Zool Ges, Gustav Fischer, Stuttgart, pp 16-33

Schnitzler HU, Flieger E (1983) Detection of oscillating target movements by echolocation in the greater horseshoe bat. J Comp Physiol 153:385-391

Schnitzler HU, Ostwald J (1982) Adaptations for the detection of fluttering insects in horseshoe bats. In: Ewert P, Capranica RR, Ingle DJ (eds) Advances in vertebrate neuroethology. Plenum Press, New York London, pp 801-827

Schnitzler HU, Menne D, Kober R, Heblich K (1983) The acoustical image of fluttering insects in echolocating bats. In: Huber F, Markl H (eds) Neuroethology and behavioral physiology. Springer, Berlin Heidelberg New York Tokyo, pp 235-250

Schuller G (1972) Echoortung bei Rhinolophus ferrumequinum mit frequenzmodulierten Lauten. Evoked potentials im Colliculus inferior. J Comp Physiol 77:306-331

Schuller G (1979) Coding of small sinusoidal frequency and amplitude modulations in the inferior colliculus of ' $\mathrm{CF}$ FM' bat, Rhinolophus ferrumequinum. Exp Brain Res 34:117-132

Schuller G, Beuter K, Schnitzler H-U (1974) Response to frequency shifted artificial echoes in the bat, Rhinolophus ferrumequinum. J Comp Physiol 89:275-286

Trappe M (1982) Verhalten und Echoortung der Grossen Hufeisennase beim Insektenfang. Dissertation, University of Tübingen, FRG

Vater M (1982) Single unit responses in cochlear nucleus of horseshoe bats to sinusoidal frequency and amplitude modulated signals. J Comp Physiol 149:369-388

Vogler B, Neuweiler G (1983) Echolocation in the noctule ( $\mathrm{Nyc}$ talus noctula) and horseshoe bat (Rhinolophus ferrumequinum). J Comp Physiol 152:421-432 